\title{
Herbal medicine of Kursi Wufarikun Ziyabit inhibits mitochondrial ATP production to activate AMPK in hepatocytes in therapy of type 2 diabetes
}

\author{
Mireguli Yasen ${ }^{1 \#}$, Lan Jiang ${ }^{1 \#}$, Mizhgona Sharofova ${ }^{2}$, Xuelei Xin ${ }^{1}$, Yusuf Nuraliev ${ }^{3}$, \\ Jianping $\mathrm{Ye}^{4^{*}}$, and Haji Akber Aisa ${ }^{1^{*}}$ \\ ${ }^{1}$ Key Laboratory of Chemistry of Plant Resources in Arid Regions, Xinjiang Technical \\ Institute of Physics and Chemistry, Chinese Academy of Science, Urumqi, Xinjiang 830011, \\ China. \\ 2 Research Center for Innovative Technologies, Academy of Sciences of Tajikistan, \\ Dushanbe 734063, Tajikistan. \\ ${ }^{3}$ Institute of Avicenna's Medicine and Pharmacology, Academy of the Tajik Traditional \\ Medicine, Dushanbe 734062, Tajikistan \\ 4 Shanghai Diabetes Institute, Shanghai Jiaotong University Affiliated Sixth People's \\ Hospital, Shanghai, China.
}

*Correspondence: Haji Akber Aisa (haji@ms.xjb.ac.cn), Jianping Ye (yejianping@sjtu.edu.cn)

\# These authors contribute equally.

\section{Word counts:}

Abstract: 292

Full text: 3548

Reference: 24

Running Title: Geranium effect on hepatocytes

Keywords: Geranium collinum, mitochondria, oxygen consumption rate, FGF21, ATP Conflict of interest: There is NO conflict of interest to disclose. All authors have read the manuscript and reached an agreement in publication of the study. The manuscript has not been submitted to other journals.

Financial support: This work was supported by the West Light Foundation of the Chinese Academy of Sciences (Grant No. 2016-QNXZ-B-4, 2018-YDYLTD-001) and the Central Asia Drug Research and Development Center of Chinese Academy of Sciences. 


\section{Abstract}

3 The herbal medicine of Kursi Wufarikun Ziyabit contains two herbs (Geranium collinum

4 Steph.ex Willd and Hypericum Scabrum Lnn) in the formula of ethnomedicine. It is usually

5 used in the treatment of diabetes mellitus with a significant therapeutic efficacy. However,

6 the molecular mechanism remains unknown for the action of medicine. In this study, we

7 investigated the mechanism with a focus on an extract (GC30) of the medicine in the

8 regulation of energy metabolism in hepatocytes. GC30 was prepared from a crude extract of

9 the medicine through elution of an AB-8 macro porous resin column loaded with the medicine in $30 \%$ ethanol. GC30 exhibited an activity in the inhibition of triglyceride (TG) accumulation in the mouse hepatocytes through a suppression of SREBP1c activity. FGF21

12 (fibroblast growth factor 21) expression was induced by GC30 in a dosage-dependent manner at concentrations of $25-100 \mu \mathrm{g} / \mathrm{ml}$. The induction was observed in mRNA and protein of FGF21, which were peaked at 2 hours and lasted for 8 hours in the response to GC30 (100 $\mu \mathrm{g} / \mathrm{ml})$. The transcription of FGF21 gene was induced by GC30 for an increase in the FGF21 gene promoter activity. AMPK and PKA activities were induced by GC30 with an elevation in their phosphorylation status, which were associated with a reduction in ATP abundance and an increase in CREB phosphorylation in cells treated with GC30. Oxygen consumption of mitochondria was inhibited in the hepatocytes by GC30. These activities of GC30 were similar to those of diabetes medicines including metformin and berberine. The data suggest that GC30 inhibited ATP production in mitochondria to activate AMPK and PKA in the hepatocytes to induce FGF21 expression. This study suggests a novel activity of the herbal medicine in the regulation of glucose and lipid metabolism in the hepatocytes. 


\section{Introduction}

25 Herbal medicines represent a resource of natural compounds in the control of blood glucose

26 in the treatment of diabetes ${ }^{[1]}$. Folk medicines provide thousands of drug candidates with

27 activities in the control of diabetes. In this case, a formula herbal medicine by the name of

28 Kursi Wufarikun Ziyabit is an ethnomedicine from Avicenna's “Canon of Medicine”, which

29 has a long history in the treatment of diabetes mellitus. The formula contains two different herbs, Geranium collinum Steph.ex Willd and Hypericum Scabrum Lnn. They are common herbs in the countries of Central Asia. Geranium collinum is a perennial plant that belongs to the family of Geraniaceae, which grows in the tropical and subtropical areas with 810 species in 8 genera in the world. Decoction of the Geranium collinum roots has a record in the treatment of the gastric diseases, postpartum bleeding, and hemoptysis as an astringent. In China, Tajikistan and Mongolia, various types of geranium are used in the treatment of hepatic and gastrointestinal diseases in the folk medicines. The water extracts of Geranium collinum roots are also used in the treatment of inflammation-related diseases, such as rheumatism and gout. Aqueous extract of the aerial part of Geranium collinum (from the flowers and leaves) are widely used in the treatment of eye diseases in Tibetan and Mongolian traditional medicine. The alcohol extract of the aerial part of Geranium collinum has a potent anti-inflammatory effect in the treatment of pneumonia, catarrhal symptoms of stomach and intestines. Geranium collinum contains phenols (pyrogallol, catechol), saponins, tannins, carbohydrates (monosaccharides, sucrose, glucose, fructose, sorbose, rhamnose, arabinose, xylose, ribose, maltose, starch, hemicellulose), vitamin C, flavonoids (avikulyarin, 
46

activity remains unknown in the treatment of diabetes mellitus. In this study, we addressed this issue by testing the effect of Kursi Wufarikun Ziyabit on hepatocytes.

AMP-activated kinase (AMPK) is a molecular target in the control of blood glucose in the type 2 diabetes ${ }^{[2]}$. AMPK is activated in the liver by the diabetic medicines, such as metformin and berberine, to reduce the fasting blood glucose. The mechanism is related to inhibition of gluconeogenesis in hepatocytes to decrease glucose production by the liver ${ }^{[3]}$. AMPK suppresses transcription-dependent expression of glucogenic genes by targeting the transcription factors (FOXO1 and CREB, etc.) ${ }^{[2]}$. AMPK inhibits de novo lipogenesis through a similar mechanism by targeting expression or phosphorylation of lipogenic proteins (SREBP and ACC, etc.) ${ }^{[2]}$. A reduction in AMPK activity leads to production of glucose by liver, which represents a mechanism for the hyperglycemia in type 2 diabetes. Activation of AMPK is a molecular mechanism for the therapeutic activities of metformin and berberine in the treatment of type 2 diabetes ${ }^{[4]}$. However, the activity of Kursi Wufarikun Ziyabit remains unknown in the regulation of AMPK.

FGF21 is a fasting-induced hepatokine that promotes energy expenditure through an action in multiple peripheral tissues including adipose tissue ${ }^{[5,6]}$. Blood FGF21 is predominantly secreted by the liver although other tissues including adipose tissue, pancreas, and skeletal muscle also produce FGF21 ${ }^{[7,8]}$. FGF21 induces beige cell functions through activation the transcription factor PGC-1 ${ }^{[5]}$. The FGF21 action is mediated by a FGF receptor complex containing $\beta$-klotho. Administration of recombinant FGF21 or transgenic overexpression of 
68 FGF21 in mice provides a protection against diet-induced obesity and glucose disorders ${ }^{[9]}$.

69 The activity of Kursi Wufarikun Ziyabit is unknown in the regulation of FGF21 expression.

71 In this study, we report that GC30 fraction of Kursi Wufarikun Ziyabit inhibited hepatic

72 steatosis and induced FGF21 expression in hepatocytes. The mechanism was associated with

73 an activation of AMPK through inhibition of ATP production in mitochondria. The study

74 provides a cellular and molecular mechanism for the pharmacological activities of Kursi

75 Wufarikun Ziyabit in the treatment of diabetes.

\section{Methods}

Plant material

The two herbs Geranium collinum Steph and Hypericum scabrum Lnn. were collected from the Botanical Garden, Academy of Sciences of the Republic of Tajikistan (38.5357500 N, 68.7790500 E and $767 \mathrm{~m}$ above sea level, Dushanbe, Tajikistan) in June 2016. The plants were identified by Qurbonov Mansur. A voucher specimen has been deposited in Xinjiang Technical Institute of Physics and Chemistry, Urumqi, Chinese Academy of Sciences. and Hypericum scabrum Lnn. (15g) in the formula of this study. The herbs were grinded into powders individually and mixed at the ratio $2.3: 1$ as a powder mixture. The mixture was extracted with $50 \%$ ethanol at 70 . The GC30 extract was obtained from a 50\% ethanol extract of the powder mixture following a purification process on an AB-8 macro porous resin column, which was washed successively with distilled water (1BV), 30\% ethanol (2BV), 
and $70 \%$ ethanol (3BV). The eluents of $30 \%$ and $70 \%$ ethanol were concentrated under

91 reduced pressure and named as named GC30 and GC70, respectively. The compounds in

92 GC30 were determined as described in supplementary material.

Cell culture and reagents

94 The mouse hepatocyte cell line Hepa-1c1c7 (1c1c7 in this study) and HepG2 cells were

95 purchased from the American Type Culture Collection (ATCC) (Manassas, VA 20110) and maintained in Dulbecco's Modified Eagle's Medium (DMEM) supplemented with 10\% fetal bovine serum at $37{ }^{\circ} \mathrm{C}$ in a $5 \% \mathrm{CO}_{2}$ incubator. Hepatic steatosis was established in HepG2 cells with a treatment by oleic acid $(200 \mu \mathrm{M})$ for $72 \mathrm{hr}$. The cells were treated with GC30 after serum-starvation in DMEM medium containing $0.25 \%$ fatty acid-free bovine serum albumin overnight. Berberine and metformin were used as positive controls in this study. Berberine (BBR, 200275,), Metformin (Met, PHR1084, Sigma-Aldrich), Geraniin (GN, E-0742, Tauto Biotech) and Ellagic acid (EA, E-0654, Tauto Biotech) were purchased from the corresponding commercial sources.

Western blot

FGF21 Protein and AMPK phosphorylation were determined in Western blot as described elsewhere ${ }^{[10]}$. The whole cell lysate was prepared in a lysis buffer with sonication as

107 described elsewhere ${ }^{[10]}$. Antibodies to FGF21 (ab171941) and actin (ab6276) were from 108 Abcam (Cambridge, MA). Antibodies to SREBP1c (sc-8984, Santa Cruz Biotech., USA), pAMPKa (Thr172, \#2531, Cell Signaling Biotech., USA) and AMPKa (sc-398861, Santa 
112 FGF21 mRNA was determined in cells using qRT-PCR. Total RNA was prepared from the 113 cells using TRIzol reagent following the manufacturer's protocol (Invitrogen, Carlsbad, CA).

114 Fgf21 mRNA was determined in mouse 1c1c7 cells with the SYBR green primer (forward 5-

115 CCTCTAGGTTTCTTTGCCAACAG-3; reverse 5-AAGCTGCAGGCCTCAGGAT-3) ${ }^{[11]}$.

116 Target mRNA was normalized to ribosome 18S RNA in qRT-PCR. SYBR Green Master Mix

117 (4309155, Applied Biosystems) was used in qRT-PCR with the ABI 7900 machine.

118 Lipids assay

119 Cells were lysed in PBS (1 g: $20 \mathrm{~mL})$. The lipids were extracted from the lysate using a

120 chloroform/methanol (2:1) mixture ${ }^{[12]}$. TG and glycerol were determined using the Serum

121 Triglyceride Determination Kit (TR0100; Sigma-Aldrich). Oil red O staining was performed

122 in HepG2 cells using a protocol described elsewhere ${ }^{[13]}$.

123 Mitochondria and ATP assays

124 Mitochondrial activity was determined in response to GC30 treatment by monitoring the 125 oxygen consumption rate (OCR) using a XF24 Analyzer (Seahorse Bioscience Inc., North 126 Billerica, MA). OCR was determined in the cells following $1 \mathrm{~h}$ pretreatment by GC30 in the 127 serum-free DMEM medium. The assay was conducted in the XF base medium (Cat \# 128 102353-100, Agilent Seahorse XF) supplemented with glucose $(25 \mathrm{mM})$, pyruvate $(1 \mathrm{mM})$ and glutamine $(4 \mathrm{mM})$ to fuel the cells. In the control, berberine $(10 \mu \mathrm{M})$ was used as a 130 positive control for mitochondrial inhibitor. Following chemicals were used at a final 131 concentration in the assay to modulate the respiratory chain: oxidative phosphorylation 132 uncoupler (Carbonyl cyanide 4-trifluoromethoxy phenylhydrazone, FCCP, $2 \mu \mathrm{M}$ ), the 133 complex V inhibitor (Oligomycin, $1 \mu \mathrm{M}$ ), complex II and III inhibitor (Antimycin A, $0.5 \mu \mathrm{M}$ ) 
134 and complex I inhibitor (Rotenone $0.5 \mu \mathrm{M}$ ). ATP production by mitochondria was 135 determined in two ways. The first was a luciferin-based ATP assay (ATP Determination Kit, 136 Life technologies, Eugene, Oregon, USA) as described elsewhere ${ }^{[14]}$ and the second was 137 determined by the value of OCR change following GC30 treatment.

FGF21 gene reporter assay

139 A FGF21 luciferase reporter (0.2 mg DNA per well) was transfected into HepG2 cells in a 140 24-well plate $\left(4 \times 10^{5}\right.$ cells per well) using Lipofectamine 2000 (Cat \#11668027, 141 ThermoFisher Scientific, Waltham, MA, USA). In the reporter vector, the luciferase gene 142 was under control of a mouse FGF21 gene promoter $(-1497 /+5)$ as described elsewhere ${ }^{[15]}$. 143 The reporter activity was determined using the dual luciferase substrate system (E1910; 144 Promega, Madison, WI, USA), and normalized with the Renilla luciferase. Each experiment 145 was repeated three times with consistent results.

\section{Statistical analysis}

147 In this study, the data were presented as the mean \pm SEM. The experiments were repeated at 148 least three times with consistent results. The representative immunoblotting is presented with 149 quantification of three blots. Student's T test was used in the data analysis with significance 150 at $p<0.05$.

\section{Results}

154 To study the metabolic activity of herbal medicine of Kursi Wufarikun Ziyabit, GC30 was 155 extracted from the powder of herb medicine and tested in hepatocytes. In the study, a hepatic 
156

steatosis model was established in the mouse $1 \mathrm{c} 1 \mathrm{c} 7$ hepatocyte cell line by treatment of the cells with oleic acid for $72 \mathrm{hr}(\mathrm{OA}, 200 \mu \mathrm{M})$ (Fig. 1A). The TG abundance was quantified in the hepatocytes using the oil red O staining and a TG assay kit. In this model, treatment of the cells with GC30 during the induction of steatosis led to a significant reduction in the TG content, and the activity was comparable to the berberine activity (Fig. 1, A-C). Berberine was used as a positive control in the inhibition of TG accumulation ${ }^{[16]}$. GC30 was used at 50 $\mu \mathrm{g} / \mathrm{ml}$ in this study. When GC30 was applied to the cells after establishment of hepatic steatosis, the GC30 activity was not observed in the reduction of TG content (Fig. 1D), suggesting that GC30 inhibits lipogenesis and does not induce lipolysis. BBR also failed to reduce TG in the established hepatic steatosis (Fig. 1D). These data suggest that GC30 inhibits TG accumulation in hepatocytes, and the mechanism is related to the inhibition of TG synthesis. The GC30 activity is comparable to that of BBR.

\section{Inhibition of SREBP1c protein by GC30}

In the hepatocytes, the transcription factor SREBP1c is one of the major driving forces in lipogenesis of hepatic steatosis. SREBP1c induces expression of multiple lipogenic enzymes through gene transcription in the induction of hepatic steatosis. SREBP1c protein was examined in the GC30-treated cells by Weston blotting to understand the mechanism of lipogenesis inhibition. In a $24 \mathrm{hr}$ study, SREBP1c was decreased in the cells by GC30 in a time-dependent manner (Fig. 2). A significant reduction was observed after $16 \mathrm{hr}$ of treatment. In the control, BBR also reduced SREBP1c protein in the model (Fig. 2). The data suggest that GC30 inhibits SREBP1c activity in hepatocytes to suppress lipogenesis in the hepatic steatosis model. 
178

179

180

181

182

183

184

185

186

187

188

189

190

191

192

193

194

195

196

197

198

199

Induction of FGF21 expression by GC30

FGF21 is a major endocrine factor from liver with an activity in the regulation of whole-body energy metabolism. FGF21 was examined in this study to determine GC30 activity in the regulation of endocrine function of hepatocytes. FGF21 protein was examined in the cell lysate by Western blotting. The protein was induced by GC30 in a dose-dependent manner and the highest activity was observed at dosages of $50-100 \mu \mathrm{g} / \mathrm{ml}$ (Fig. 3A). In the timedependent study, FGF21 was induced by GC30 during $1-24 \mathrm{hr}$. The induction was peaked at $4 \mathrm{hr}$ (Fig. 3B). These results suggest that GC30 may induce FGF21 expression in hepatocytes. Induction of FGF21 gene promoter by GC30

FGF21 expression is regulated at the transcriptional level. mRNA and the gene promoter were examined to understand the mechanism of GC30 activity. An increase in mRNA was observed in FGF21 in response to GC30 treatment in dose- and time-dependent manners (Fig.

4, A and B). The gene promoter activity was examined using a luciferase reporter controlled by the FGF21 promoter DNA ${ }^{[15]}$. The reporter activity was induced by GC30 with $200 \%$ elevation over the control (Fig. 4C). In the positive control, the reporter activity was induced by Forskolin through activation of cAMP/PKA pathway (Fig. 4D). The GC30 activity was examined in the regulation of $\mathrm{cAMP} / \mathrm{PKA}$ pathway with phosphorylation of the transcription factor CREB. An increase was observed in the phosphorylation in GC30-treated cells (Fig. 4E). The GC30 activity was similar to that of Forskolin (Fig. 4E). These results suggest that GC30 may induce FGF21 transcription in hepatocytes through activation of the cAMP/PKA signaling pathway.

Activation of AMPK and inhibition of ATP by GC30 
AMPK is an energy sensor in cell whose activity is induced by a reduction of the intracellular

201 ATP level. AMPK activity was examined to understand the mechanism of GC30 activity in 202 the inhibition of hepatic steatosis. GC30 activity was examined in the regulation of AMPK activity by the phosphorylation status (pAMPK) in the cells. The pAMPK signal was induced in the cells treated by GC30 (Fig. 5A). BBR and metformin were used as the positive controls in the activation of AMPK (Fig. 5A). The GC30 activity in the activation of AMPK was not as strong as those of BBR or Metformin. In the study, GC30 was compared with another extract of Kursi Wufarikun Ziyabit (GC70), and other compounds including GN (Geraniin) and EA (Ellagic acid). The pAMPK signal was induced by all of them (Fig. 5A). The data suggest that GC30 activated AMPK in the hepatocytes. Intracellular ATP was determined in the cells to understand the mechanism of AMPK activation by GC30. The ATP content was decrease by GC30, which was similar to those of other compounds including GC70, GN, EA, BBR and Metformin (Fig. 5B). These data suggest that GC30 may activate AMPK through reduction of ATP level in the cells.

Inhibition of mitochondrial ATP production by GC30

215 Mitochondria are the major source of ATP in hepatocytes. Mitochondrial function was examined to understand the ATP reduction in hepatocytes by GC30. The oxygen consumption rate (OCR) is an indicter of mitochondrial function as the mitochondrial activity

218 is dependent on oxygen. OCR was measured in the GC30-treated cells and a significant 219 reduction was observed in the basal and maximal (uncoupling) conditions (Fig. 6A). The ATP production is catalyzed by ATP synthase in mitochondria. Inhibition of the enzyme by oligomycin leads to a decrease in OCR. The difference of OCR at the basal and oligomycin- 
treated conditions represents the ATP production capacity in mitochondria. Oxygen is also used in the heat production (uncoupling) by mitochondria. In response to the chemical uncoupler FCCP, OCR is induced for the maximal capacity of oxygen consumption in mitochondria. The difference between the basal and maximal OCR represents the spare capacity of mitochondria. ATP production and spare capacity were all decreased in the hepatocytes by GC30 (Fig. 6B). In the study, BBR was used as the positive control. The

GC30 activity was similar to that of BBR in this assay (Fig. 6B). These data suggest that

GC30 inhibited ATP production by mitochondria in the hepatocytes.

GC30 was analyzed with liquid chromatograph-mass spectrometry to determine the in the fingerprints of GC30. Those include Quercetin, Quercetin derivative and others.

Quercetin provides a basis for certain activities of GC30 in the regulation of ATP production.

\section{5}

\section{Discussion \& Conclusions}

Our data demonstrates that the GC30 extract of herbal medicine of Kursi Wufarikun Ziyabit is able to inhibit hepatic steatosis and induce FGF21 expression in hepatocytes. The medicine has been used for hundreds of years in the treatment of diabetes and liver diseases in forks medicines of the countries of Central Asia. However, the cellular and molecular mechanisms were not known for the therapeutic activities. To address this issue, we prepared the GC30 extract of Kursi Wufarikun Ziyabit, and tested its activity in the hepatocyte model. GC30 was found to have activities in the inhibition of development of hepatic steatosis and induction of FGF21 expression in the hepatocyte model. The data suggest that liver is a potential target of 
244 the herbal medicine in the treatment of diabetes and liver disease. The mechanism is related

245 to improvement of hepatocyte function by inhibition of steatosis and induction of the 246 endocrine activity. These observations provide a cellular mechanism for the therapeutic 247 activities of the herbal medicine.

Our data suggests that in hepatocytes, GC30 activated the AMPK and cAMP/PKA signaling 250 pathways to generate the pharmacological activities. The AMPK pathway provides a 251 mechanism for inhibition of hepatic steatosis. In cells, AMPK is activated by lack of fuel supply in the stress conditions. AMPK activation acts to restore the fuel supply in cells

253 through an impact in multiple metabolic pathways ${ }^{[2]}$. Inhibition of the de novo lipogenic pathway by AMPK shifts energy metabolism from anabolism to catabolism, which includes induction of utilization of glucose and fatty acids in the production of ATP. AMPK activation by GC30 represents a molecular mechanism for the GC30 activity in the inhibition of hepatic steatosis. The cAMP/PKA pathway stimulates fuel mobilization and utilization in ATP production through stimulation of glycose and fatty acid oxidation in mitochondria. It also induces FGF21 expression in hepatocytes. Those activities support another molecular mechanism of the GC30 activity in the inhibition of hepatic steatosis. Although the cAMP/PKA pathway induces gluconeogenesis in hepatocytes, the activity may be offset by 262 the activation of AMPK in the GC30-treated cells. AMPK is known to inhibit 263 gluconeogenesis in the liver. Activation of the two pathways (AMPK and cAMP/PKA) provides two molecular mechanisms for the activities of GC30 in the regulation of hepatocyte metabolism. 
267 GC30 exhibited an activity in the suppression of ATP production in mitochondria, which was

268 similar to those of BBR and metformin. BBR and metformin are widely used in the control of

269 blood glucose in the diabetic patients worldwide. They share an activity in the activation of

270 the AMPK signaling pathway by inhibition of ATP production in mitochondria ${ }^{[17]}$. In current

271 study, GC30 was found to reduce the intracellular level of ATP by suppression of

272 mitochondrial oxygen consumption, which provides a mechanism for the activation of

273 AMPK and cAMP/PKA pathways. Activation of the two pathways is likely a compensatory

274 response to the ATP depletion in favor of ATP production. A similar reaction was reported in

275 our previous study of BBR, which inhibited ATP production by mitochondria with a

276 reduction in oxygen consumption ${ }^{[18]}$. Interestingly, such an activity was also reported for

277 metformin by other group ${ }^{[19]}$. Consistently, ATP enhances de novo lipogenesis and

278 gluconeogenesis in liver in the pathogenesis of insulin resistance ${ }^{[20,21]}$. Inhibition of ATP

279 production by an uncoupler (2,4 dinitrophenol, DNP) prevented insulin resistance in dietary

280 obese rats ${ }^{[21]}$. Elevation of intracellular ATP promotes insulin resistance and inflammation in

281 previous studies by us ${ }^{[14,22]}$. Suppression of ATP production by mitochondria provides a

282 cellular basis for the GC30 activities in the activation of AMPK in hepatocytes. The

283 molecular target of GC30 remains to be identified in mitochondria.

285 The compound profile was examined in GC30 using the liquid chromatography-mass spectrometry (LC-MS) technology. Twenty compounds were identified in GC30, in which quercetin and its derivatives were found. Quercetin has an activity in the inhibition of 
288 mitochondrial function to decrease ATP production ${ }^{[23]}$, which may contribute to the GC30 289 activity in the regulation of AMPK. Quercetin inhibits lipogenesis in adipocytes ${ }^{[24]}$, which is 290 consistent with the GC30 activity in the inhibition of lipogenesis in hepatocytes.

In summary, we investigated the activity of herbal medicine of Kursi Wufarikun Ziyabit in the regulation of hepatocyte metabolism in this study. The study was conducted with the GC30 extract in hepatocytes. GC30 inhibited the development of hepatic steatosis and induced expression of FGF21 through a transcriptional mechanism in hepatocytes. The activities were associated with activation of the AMPK and cAMP/PKA pathways. GC30 inhibited ATP production in mitochondria to activate the two signaling pathways through was identified in GC30 together with other 19 compounds to support the mechanism of GC30 activities. The study suggests that the herbal medicine may generate the therapeutic activities by targeting liver. However, this possibility remains to be tested in vivo.

\section{Acknowledgments}

This work was supported by the West Light Foundation of the Chinese Academy of Sciences

(Grant No. 2016-QNXZ-B-4, 2018-YDYLTD-001) and the Central Asia Drug Research and

Development Center of Chinese Academy of Sciences.

Author contribution: MY, LJ, XX, MS and YN conducted the experiments and analyzed the 
manuscript. HAA is the guarantor of this work and has full access to all the data in the study

311 and takes responsibility for the integrity of the data and accuracy of the data analysis. All

312 authors reviewed the results and approved the final version of the manuscript. There is NO

313 conflict of interest to disclose.

314

315 
317 Fig. 1 Inhibition of TG accumulation by GC30 in HepG2 cells. HepG2 cells were treated 318 with oleic acid $(200 \mu \mathrm{M})$ for 72 hours to induce TG accumulation in the hepatic steatosis model. GC30 was used at $50 \mu \mathrm{g} / \mathrm{ml}$ in this study. A. TG detection with oil red $\mathrm{O}$ staining. Lipid droplets are indicated by the red color in the HepG2 cell cytoplasm following oil red $\mathrm{O}$ staining. GC30 was applied to the cells together with the oleic acid during induction of TG accumulation. B. TG quantification by OD of color change. OD was determined in the extract of hepatocytes for oil red O. C. TG quantification by the TG assay kit. TG was extracted and quantified with the TG assay kit. D. GC30 treatment of established steatosis. In the bar figure, the results are mean $\pm \operatorname{SE}(n=3) .{ }^{*} \mathrm{p}<0.05$; $^{\#} \mathrm{p}<0.05$ over the control. protein was examined in HepG2 hepatocytes after GC30 $(100 \mu \mathrm{g} / \mathrm{ml})$ or BBR $(10 \mu \mathrm{M})$ control.

Fig. 3 Induction of FGF21 expression in hepatocytes by GC30. FGF21 expression was examined in 1c1c7 hepatocytes after GC30 treatment. A. FGF21 protein expression. The whole cell lysate was used in the Western blot for FGF21 protein. The dose-dependent study was done with GC30 treatment for $4 \mathrm{hr}$. B. FGF21 protein in a time-course study. GC30 (100 $\mu \mathrm{g} / \mathrm{ml})$ was used to treat the cells for $24 \mathrm{hr}$. The bar figure represents mean $\pm \mathrm{SE}(\mathrm{n}=3) .{ }^{*} \mathrm{p}<$ $0.05 ;{ }^{* *} \mathrm{p}<0.01 ;{ }^{* * *} \mathrm{p}<0.001$ over the control.

Fig. 4 Activation of FGF21 gene transcription by GC30. A. Induction of FGF21 mRNA 337 by GC30 in the dose-dependent study. The FGF21 mRNA was determined in 1c1c7 cells 
following a $2 \mathrm{hr}$ treatment with different dosages of GC30. mRNA was determined using qRT-PCR. B. Induction of FGF21 mRNA by GC30 in the time-dependent study. The FGF21 mRNA was determined in 1c1c7 cells with GC30 $(100 \mu \mathrm{g} / \mathrm{ml})$ treatment over $24 \mathrm{hr}$. C. Induction of the FGF21 luciferase reporter by GC30. The FGF21 reporter was transiently transfected into 293 cells, and the cells were treated with GC30 (50 $\mu \mathrm{g} / \mathrm{ml})$ for $16 \mathrm{hr}$. D. Induction of the FGF21 gene promoter activity by Forskolin. The reporter activity was determined after Forskolin $(10 \mu \mathrm{M})$ treatment of the transfected cells. E. Induction of CREB phosphorylation by GC30. The phosphorylation was determined in whole cell lysate by

Western blotting after treatment of the cells with GC30 $(50 \mu \mathrm{g} / \mathrm{ml})$ and Forskolin $(10 \mu \mathrm{M})$. In the bar figure, the results are mean $\pm \mathrm{SE}(\mathrm{n}=3) .{ }^{*} \mathrm{p}<0.05 ;{ }^{* *} \mathrm{p}<0.01 ;{ }^{* * *} \mathrm{p}<0.001$ over the control.

Fig. 5 Induction of AMPK phosphorylation and inhibition of ATP by GC30. A. Induction of AMPK phosphorylation by GC30. The HepG2 cells were treated with GC30. exposure to GC30 $(50 \mu \mathrm{g} / \mathrm{ml}), \mathrm{GC} 70(50 \mu \mathrm{g} / \mathrm{ml})$, GN $(10 \mu \mathrm{M})$, and EA $(10 \mu \mathrm{M})$ by Western blotting. BBR $(10 \mu \mathrm{M})$ and Met $(10 \mu \mathrm{M})$ was in the positive controls as AMPK activators. B. Inhibition of ATP by GC30. ATP was determined in the whole cell lysate after GC30 treatment of HepG 2 cells. The fold change of ATP is presented in the unit of $\mu \mathrm{M} / \mathrm{g}$ protein. In the bar figure, the results are mean $\pm \mathrm{SE}(\mathrm{n}=3) .{ }^{*} \mathrm{p}<0.05 ;{ }^{* *} \mathrm{p}<0.01$ over the control.

Fig. 6 Inhibition of mitochondrial ATP production by GC30. A. Dynamic change of in the HepG2 cells following GC30 $(50 \mu \mathrm{g} / \mathrm{ml})$ for $1 \mathrm{hr}$. In the positive control, BBR $(10 \mu \mathrm{M})$ 

production. The value was a result of OCR difference before and after oligomycin treatment. the control. 


\section{References}

[1] Yin J, Zhang H, Ye J. Traditional chinese medicine in treatment of metabolic syndrome. Endocr Metab Immune Disord Drug Targets 2008; 8:99-111.

[2] Garcia D, Shaw RJ. Ampk: Mechanisms of cellular energy sensing and restoration of metabolic balance. Mol Cell 2017; 66:789-800.

[3] Ye J. Mechanisms of insulin resistance in obesity. Frontiers of medicine 2013; 7:14-24.

[4] Yin J, Ye J, Jia W. Effects and mechanisms of berberine in diabetes treatment. Acta Pharmaceutica Sinica B 2012; 2:327-334.

[5] Fisher FM, Kleiner S, Douris N, et al. Fgf21 regulates pgc-1alpha and browning of white adipose tissues in adaptive thermogenesis. Genes Dev 2012; 26:271-281.

[6] Veniant MM, Hale C, Helmering J, et al. Fgf21 promotes metabolic homeostasis via white adipose and leptin in mice. PLoS One 2012; 7:e40164.

[7] Potthoff MJ, Kliewer SA, Mangelsdorf DJ. Endocrine fibroblast growth factors 15/19 and 21: From feast to famine. Genes Dev 2012; 26:312-324.

[8] Markan KR, Naber MC, Ameka MK, et al. Circulating fgf21 is liver derived and enhances glucose uptake during refeeding and overfeeding. Diabetes 2014; 63:4057-4063.

[9] Kharitonenkov A, Shiyanova TL, Koester A, et al. Fgf-21 as a novel metabolic regulator. J Clin Invest 2005; 115:1627-1635.

[10] Xu F, Gao Z, Zhang J, et al. Lack of sirt1 (mammalian sirtuin 1) activity leads to liver steatosis in the sirt1+/mice: A role of lipid mobilization and inflammation. Endocrinology 2010; 151 2504-2514.

[11] Inagaki T, Dutchak P, Zhao G, et al. Endocrine regulation of the fasting response by ppar[alpha]-mediated induction of fibroblast growth factor 21. Cell Metabolism 2007; 5:415-425.

[12] Lin J, Yang R, Tarr PT, et al. Hyperlipidemic effects of dietary saturated fats mediated through pgc-1beta coactivation of srebp. Cell 2005; 120:261-273.

[13] Lu H, Gao Z, Zhao Z, Weng J, J Y. Transient hypoxia reprograms differentiating adipocytes for enhanced insulin sensitivity and triglyceride accumulation Int J Obes 2016; 40:121-128.

[14] Zhang Y, Zhao Z, Ke B, et al. Induction of posttranslational modifications of mitochondrial proteins by atp contributes to negative regulation of mitochondrial function. PLoS One 2016; 11:e0150454.

[15] Li H, Gao Z, Zhang J, et al. Sodium butyrate stimulates expression of fibroblast growth factor 21 in liver by inhibition of histone deacetylase 3. Diabetes 2012; 61:797-806.

[16] Xia X, Yan J, Shen Y, et al. Berberine improves glucose metabolism in diabetic rats by inhibition of hepatic gluconeogenesis. PLoS One 2011; 6:e16556.

[17] Zhang Y, Ye J. Mitochondrial inhibitor as a new class of insulin sensitizer. Acta Pharmaceutica Sinica B 2012; 4:341-349.

[18] Yin J, Gao Z, Liu D, Liu Z, Ye J. Berberine improves glucose metabolism through induction of glycolysis. Am J Physiol Endocrinol Metab 2008; 294:E148-E156.

[19] Owen MR, Doran E, Halestrap AP. Evidence that metformin exerts its anti-diabetic effects through inhibition of complex 1 of the mitochondrial respiratory chain. Biochem J 2000; 348 Pt 3:607-614.

[20] Satapati S, Kucejova B, Duarte JA, et al. Mitochondrial metabolism mediates oxidative stress and inflammation in fatty liver. J Clin Invest 2015; 125:4447-4462.

[21] Perry RJ, Zhang D, Zhang XM, Boyer JL, Shulman GI. Controlled-release mitochondrial protonophore reverses diabetes and steatohepatitis in rats. Science 2015; 347:1253-1256.

[22] Lee JH, Zhang Y, Zhao Z, et al. Intracellular atp in balance of pro- and anti-inflammatory cytokines in 
bioRxiv preprint doi: https://doi.org/10.1101/2020.09.28.316349; this version posted September 29, 2020. The copyright holder for this preprint (which was not certified by peer review) is the author/funder. All rights reserved. No reuse allowed without permission.

411 adipose tissue with and without tissue expansion. Int J Obes (Lond) 2017; 41:645-651

412 [23] Hawley SA, Ross FA, Chevtzoff $C$, et al. Use of cells expressing gamma subunit variants to identify diverse 413 mechanisms of ampk activation. Cell Metab 2010; 11:554-565.

414 [24] Ahn J, Lee H, Kim S, Park J, Ha T. The anti-obesity effect of quercetin is mediated by the ampk and mapk 415 signaling pathways. Biochem Biophys Res Commun 2008; 373:545-549.

416 
Fig. 1 Inhibition of TG accumulation in hepatocytes by GC30

A. Oil red $O$ staining

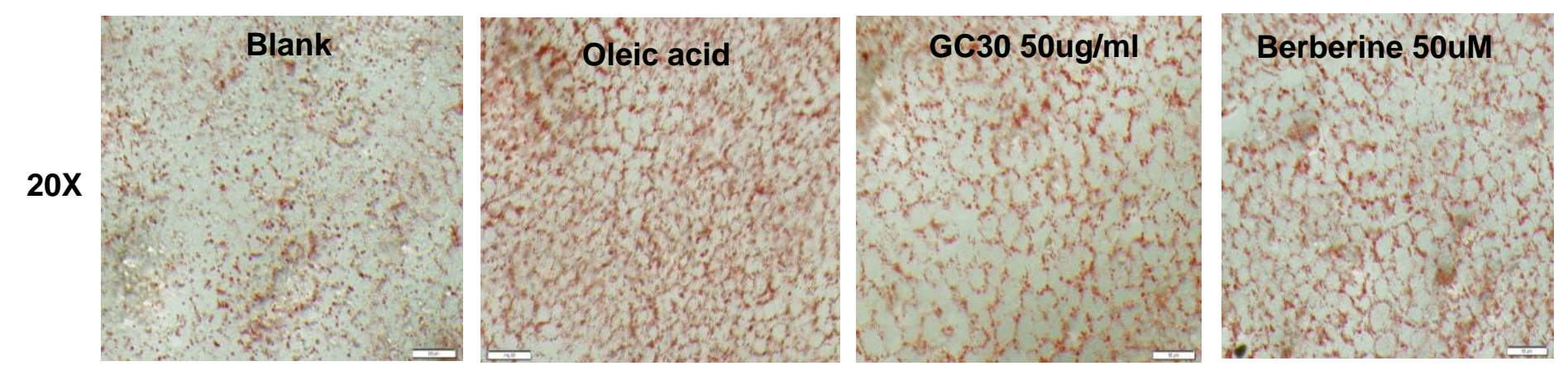

B. Quantification of oil red O

C. TG in cell lysate

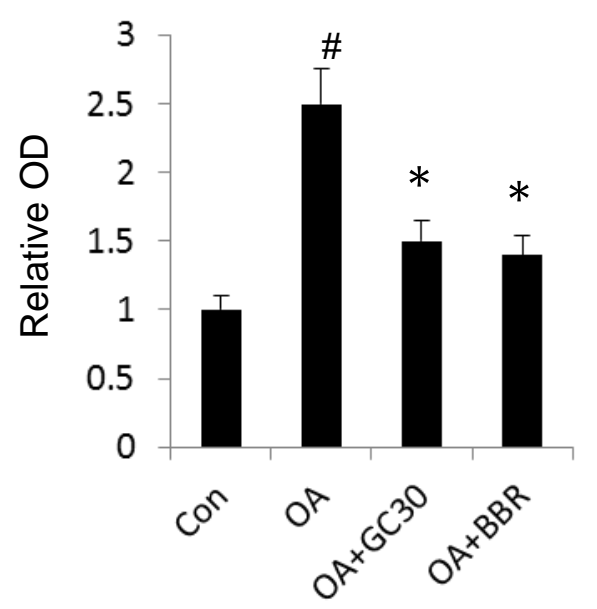

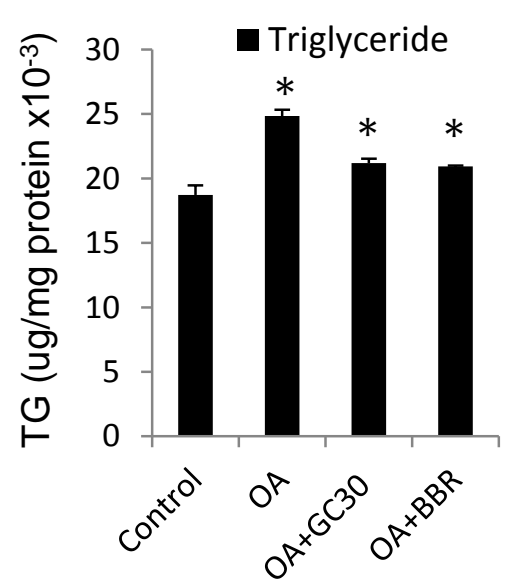

D.
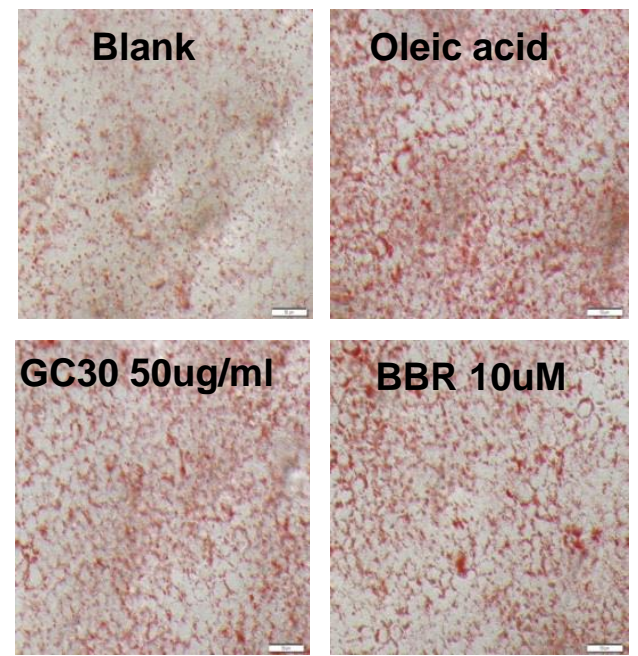

$20 X$ 
Fig. 2 Inhibition of SREBP in hepatocytes by GC30

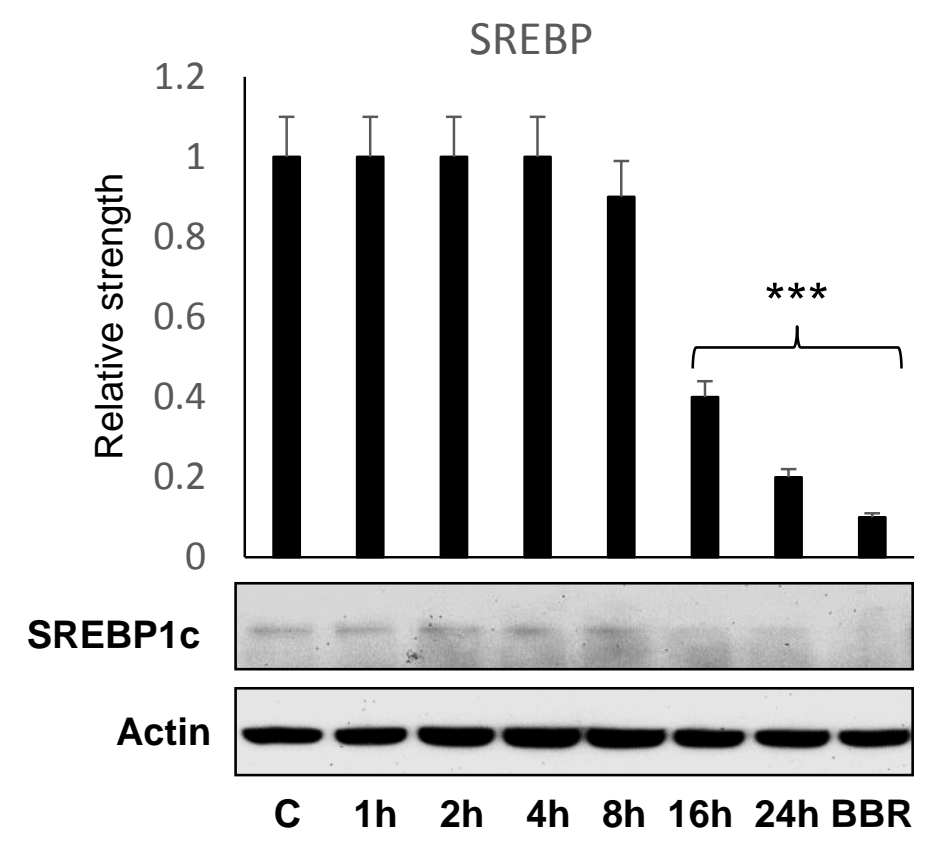


Fig. 3 Induction of FGF21 expression by GC30 in 1c1c7 cells

A. GC30 dose-dependent effcet

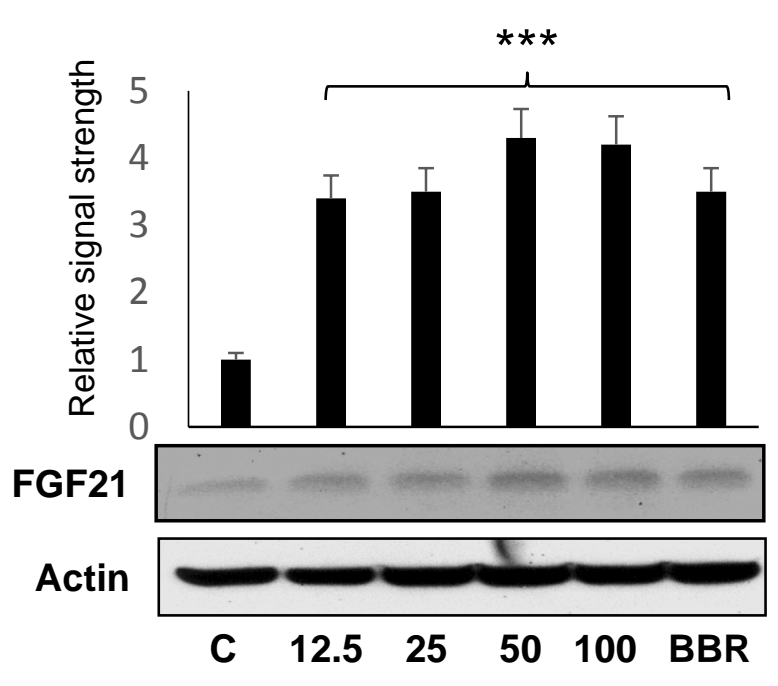

B. GC30 time-dependent effect

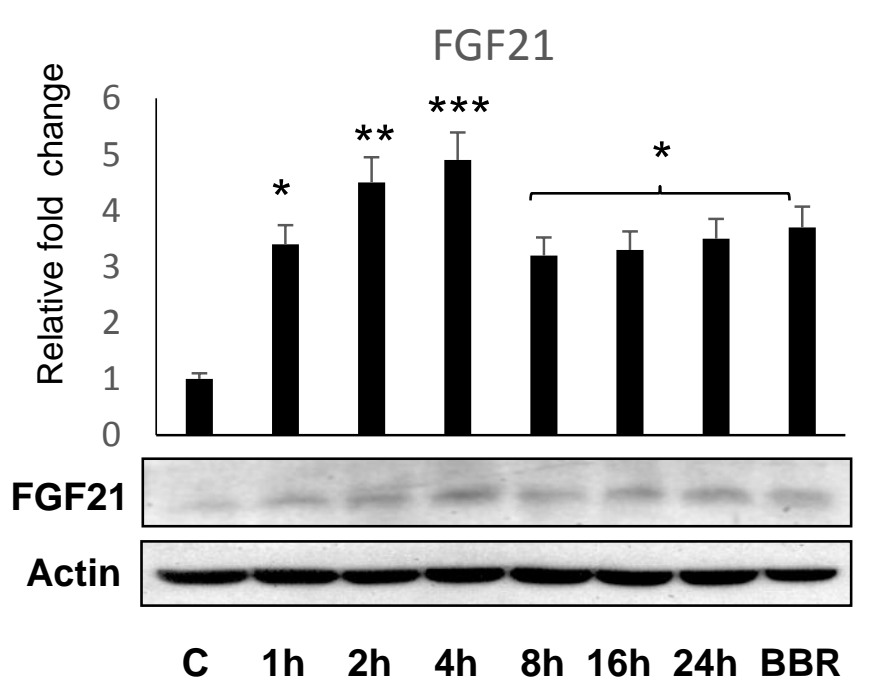


A. GC30 dose-dependent effect

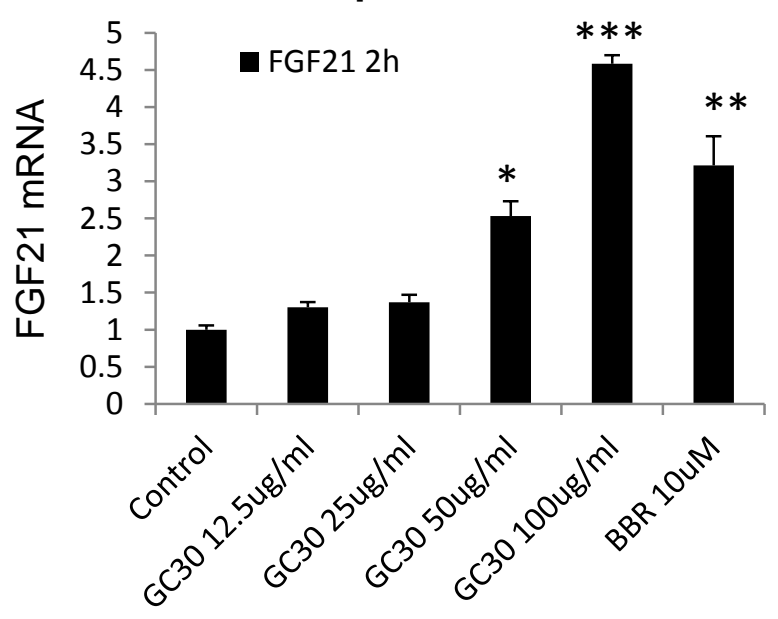

B. GC30 time-dependent effect

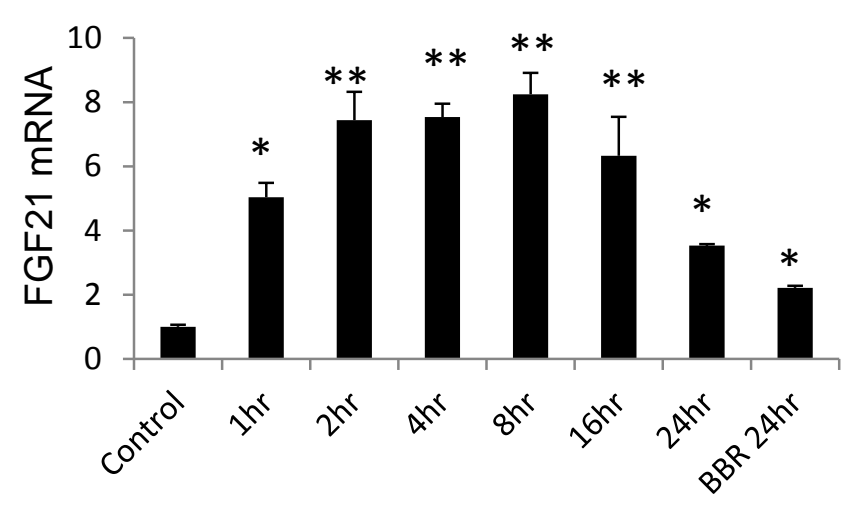

C. GC30 effect

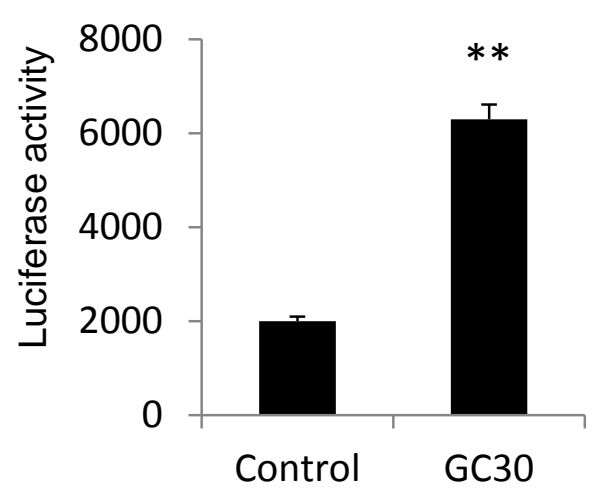

D. cAMP effect

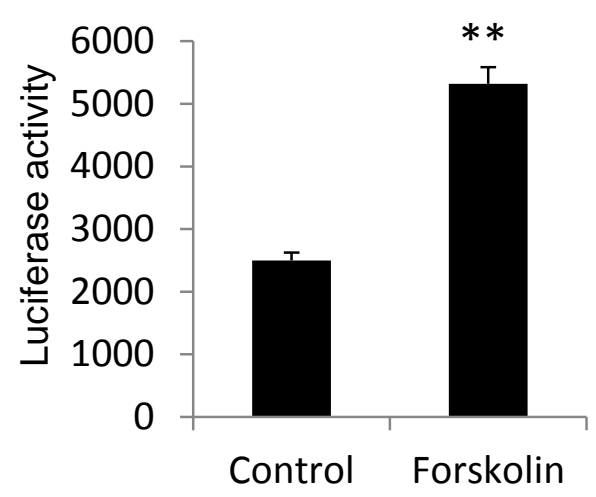

E. pCREB signal

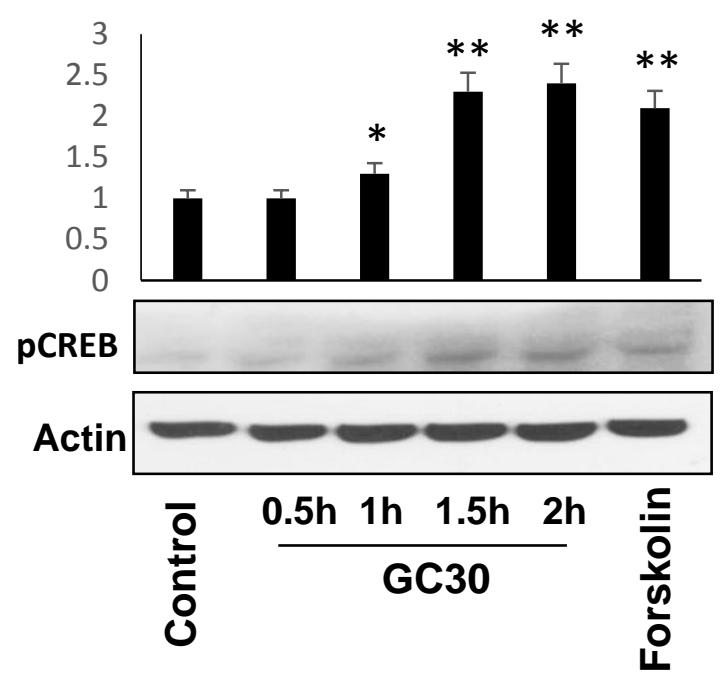


Fig. 5 Induction of AMPK phosphorylation and inhibition of ATP by GC30

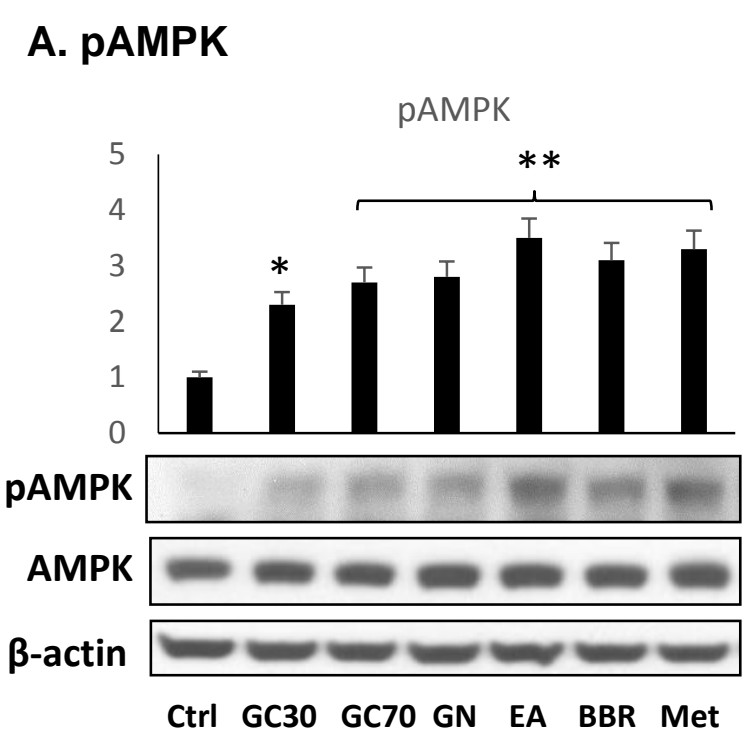

B. ATP concentration

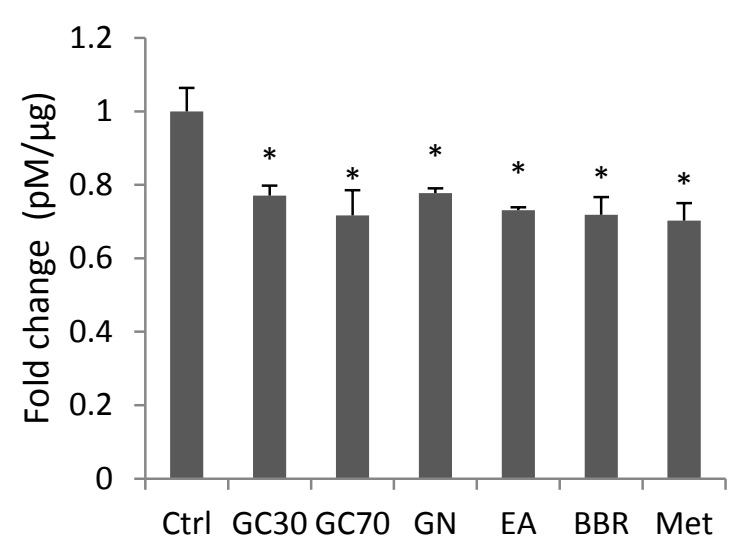




\section{A. Oxygen consumption}

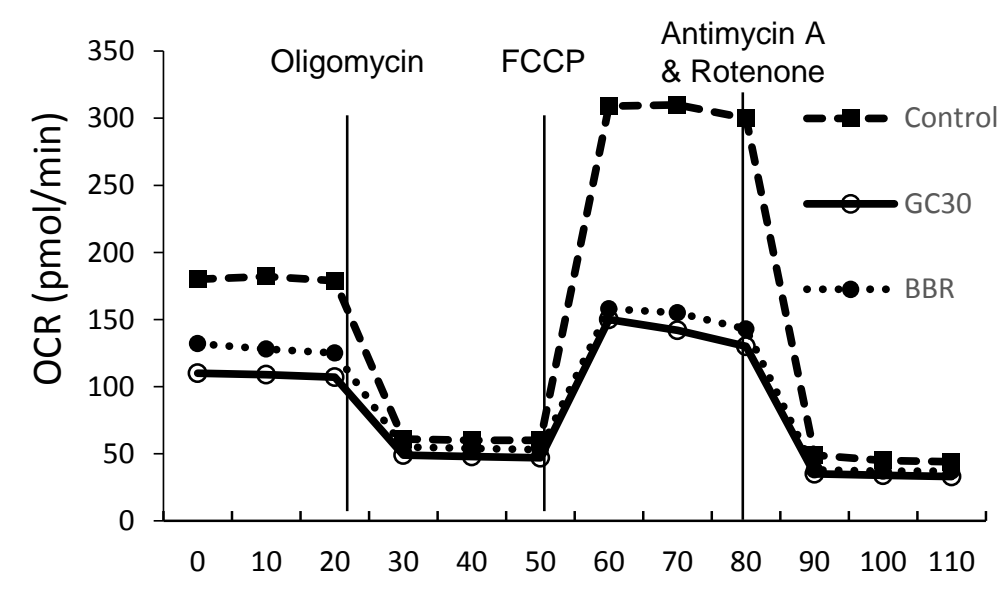

B. ATP production

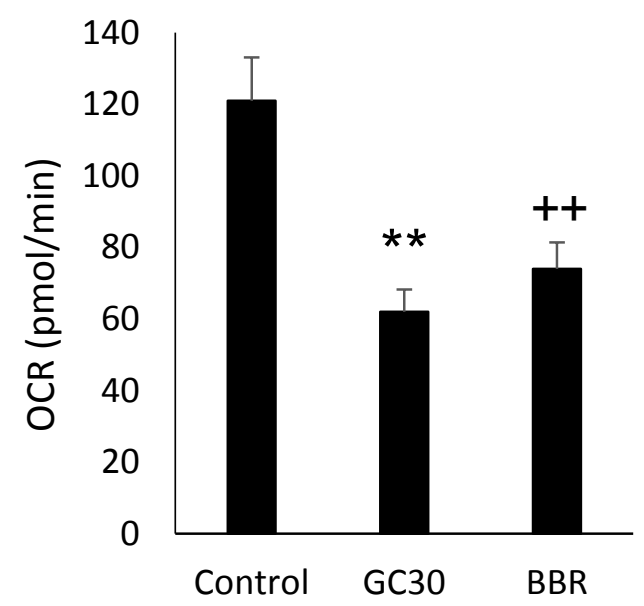

C. Spare capacity

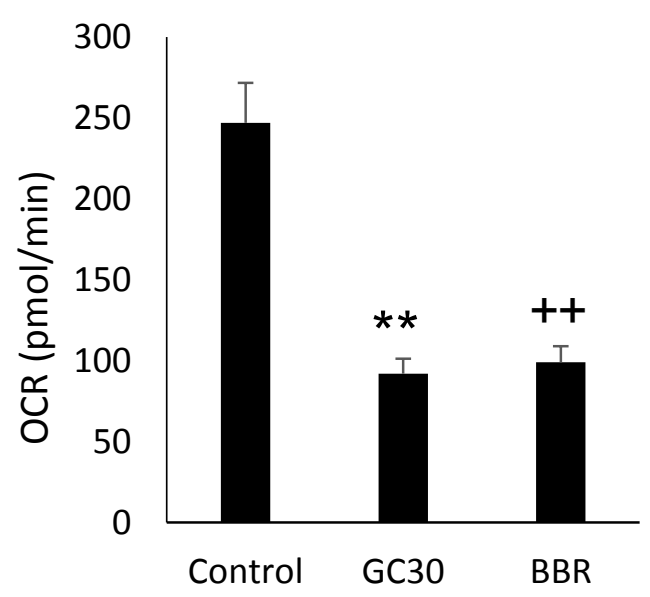

